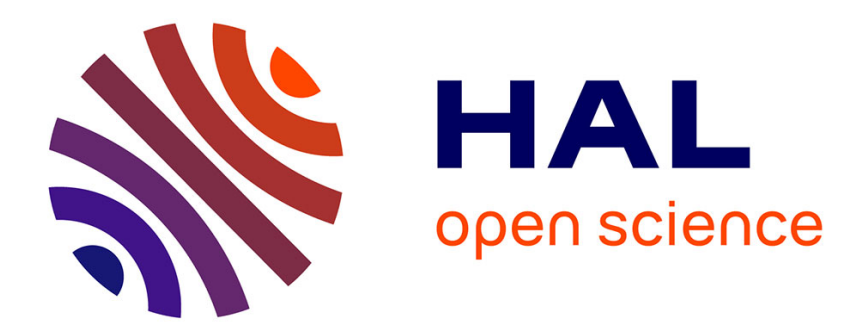

\title{
Différenciation rapide des colonies de colibacilles parmi les colonies de coliformes
}

\author{
J. Jacquet, O. Coiffier
}

\section{To cite this version:}

J. Jacquet, O. Coiffier. Différenciation rapide des colonies de colibacilles parmi les colonies de coliformes. Le Lait, 1978, 58 (573_574), pp.111-117. hal-00928785

\section{HAL Id: hal-00928785 \\ https://hal.science/hal-00928785}

Submitted on 1 Jan 1978

HAL is a multi-disciplinary open access archive for the deposit and dissemination of scientific research documents, whether they are published or not. The documents may come from teaching and research institutions in France or abroad, or from public or private research centers.
L'archive ouverte pluridisciplinaire HAL, est destinée au dépôt et à la diffusion de documents scientifiques de niveau recherche, publiés ou non, émanant des établissements d'enseignement et de recherche français ou étrangers, des laboratoires publics ou privés. 


\title{
Différenciation rapide des colonies de colibacilles parmi les colonies de coliformes
}

\author{
par \\ J. JACQUET et O. COIFFIER
}

\section{S u m m a r y}

\begin{abstract}
A new test is proposed to differenciate $E$. coli from other coliforms.

Colonies from selective plates were subcultured in a peptone water for indol test and in a malonate broth, incubated at $37^{\circ} \mathrm{C}$ for $24 \mathrm{hr}$. The results were compared to Mackenzie, Gilbert and Taylor's for 637 strains sampled in 2666 coliforms isolated from several types of each of the following: cheeses, milk, cream, seawater, streams, sewage and stool specimens of calves and chickens. The particular interest of the experiment is that it allows a very precise distinction between $E$. coli and $K$. pneumoniae var. oxytoca.
\end{abstract}

\section{INTRODUCTION : BUT PROPOSE}

Dans les examens des produits laitiers, on a souvent à sa disposition des colonies obtenues sur milieux solides divers, et que l'on suppose appartenir au groupe des coliformes, non régulier du point de vue taxonomique, mais auquel les professionnels et hygiénistes sont fidèles.

Bien que la tendance actuelle s'attache plutôt, et ceci d'une façon générale en bactériologie alimentaire, à la notion de coliformes fécaux, ou même d'entérobactéries totales, il peut être important de repérer facilement les Escherichia coli parmi les autres, que l'on croit, ou non, selon les circonstances, à leur primauté comme témoins de contamination d'origine intestinale. Certains tests comme ceux de " haute température " comparés par Oger et Leclerc [9] ont déjà été proposés dans ce but. La possession d'un moyen simple et fidèle permettrait d'apporter des précisions supplémentaires et de mieux interpréter les documents analytiques dont on dispose déjà au moment où on met cette méthode en œuvre. La technique la plus 
utilisée dans ce but, celle de Mackenzie et al. [7], qui consiste en l'ensemencement couplé d'un tube d'eau peptonée, et d'un bouillon bilié lactosé au vert brillant, incubés, l'un et l'autre, à $44^{\circ} \mathrm{C}$, constitue une épreuve satisfaisante, dans l'ensemble, mais à la condition formelle d'être réalisée dans des conditions extrêmement strictes de température : l'emploi d'un bain-marie muni d'un thermostat très sensible est indispensable et la simple mise à l'étuve est exclue. En dehors de cette première difficulté d'ordre matériel, il existe, aussi, des erreurs inhérentes au comportement particulier de certains coliformes vis-à-vis de la température. Il y a, d'abord, des erreurs par excès ; mais, que l'on peut éviter, par l'obligation absolue de maintenir le degré thermique prévu : au-dessous de $44^{\circ} \mathrm{C}$, et surtout à partir de $42^{\circ} \mathrm{C}$, des espèces comme Klebsiella oxytoca donnent des gaz en bouillon bilié et de l'indole en eau peptonée, exactement comme les Escherichia coli. Or, ce biotype indologène de K. pneumoniae paraît de plus en plus fréquemment rencontré dans les produits laitiers, comme dans l'intestin des animaux ; il peut même être dominant, ainsi que nous l'avons observé dans la flore fécale de poulets de Kabylie [4].

Bien plus, des réactions faussement négatives, et impossibles à éviter, même avec des précautions techniques strictement appliquées, existent aussi. Nous avons constaté, en effet, sur 200 souches de colibacilles, que 8 p. 100 étaient thermosensibles et inhibées à des températures supérieures à $42^{\circ} \mathrm{C}-44^{\circ} \mathrm{C}$ où l'on fait justement et classiquement beaucoup de recherches de ces entérobactéries, et que l'on prend bien soin de ne pas dépasser. A $40,5^{\circ} \mathrm{C}$ par rapport à $37^{\circ} \mathrm{C} \pm 0,5^{\circ} \mathrm{C}$ nous avons obtenu comparativement, les mêmes résultats. Il n'en est plus de même entre $37^{\circ} \mathrm{C}$ (ou $40,5^{\circ} \mathrm{C}$ ) $\pm 0,5^{\circ} \mathrm{C}$ d'une part et $44^{\circ} \mathrm{C} \pm 0,5^{\circ} \mathrm{C}$, d'autre part. De leur côté, Mehlman et al., sur un total de 175 cultures en retrouvent 98 p. 100 à $42^{\circ} \mathrm{C} ; 89$ p. 100 à $44^{\circ} \mathrm{C} ; 82$ p. 100 à $44,5^{\circ} \mathrm{C}$ et 65 p. 100 seulement à $45,5^{\circ} \mathrm{C}$. La fragilité, dans cette zone, à une faible variation de chaleur apparaît très grande.

Il semble donc opportun, de remplacer le test de Mackenzie par quelque chose de plus simple et plus fidèle, n'exigeant pas, de surcroît, un équipement supplémentaire, donc réalisable à l'étuve à $37^{\circ} \mathrm{C}$.

\section{MATERIEL DISPONIBLE}

Nous disposions d'un ensemble de 2666 souches d'origines diverses (lait, crèmes fermières, fromages, feces de veaux et de poulets, eaux de mer, eaux d'égout, sols) ; 1680 provenaient du lait et des produits laitiers, soit 63,42 p. 100 de l'échantillon total, et 1599 sortaient de fromages, dont la majorité affinés au froid, ce qui explique 
la grande proportion d'Enterobacter hafniae* (971 sur 1599), qui sont relativement psychrotrophes. La répartition en est représentée dans le tableau 1.

\section{TABLEAU 1}

Répartition des espèces de coliformes étudiées

\begin{tabular}{l|c|c}
\hline \multicolumn{1}{c|}{ Espèces } & $\begin{array}{c}\text { Nombre de souches } \\
\text { isolées }\end{array}$ & $\begin{array}{c}\text { Pourcentage de souches } \\
\text { isolées par rapport } \\
\text { au nombre total }\end{array}$ \\
\hline E. coli & 1050 & 39,38 p. 100 \\
Enterobacter hafniae & 979 & 36,72 p. 100 \\
K. oxytoca & 177 & 6,63 p. 100 \\
Citrobacter & 163 & 6,11 p. 100 \\
K. pneumoniae & 147 & $5,51 \mathrm{p} .100$ \\
E. cloacae & 111 & $4,16 \mathrm{p} .100$ \\
E. coli non indologène & 12 & $0,45 \mathrm{p} .100$ \\
Klebsiella sp. & 5 & $0,18 \mathrm{p} .100$ \\
E. agglomerans & 6 & $0,22 \mathrm{p} .100$ \\
K. ozenae & 4 & $0,15 \mathrm{p} .100$ \\
Dispar & 2 & $0,075 \mathrm{p} .100$ \\
Levinea amalonatica & 1 & $0,037 \mathrm{p} .100$ \\
& & \\
\hline
\end{tabular}

\section{EPREUVES EFFECTUEES}

Nous avons choisi 637 cultures, en prenant la précaution de faire un très large éventail de chimiotypes**. Nous avons effectué simultanément, par incubation à $37^{\circ} \mathrm{C}$, les tests de production d'indole en eau peptonée, de dégagement de gaz en bouillon lactosé bilié au vert brillant et d'utilisation du malonate de sodium. Nos résultats sont résumés dans le tableau 2. On y voit que la production de gaz

$*$ Hafniae $=$ E. alvei.

** Identification selon l” "API SYSTEM ». 
TABLEAU 2. - Répartition des résultats selon les variétés de coliformes

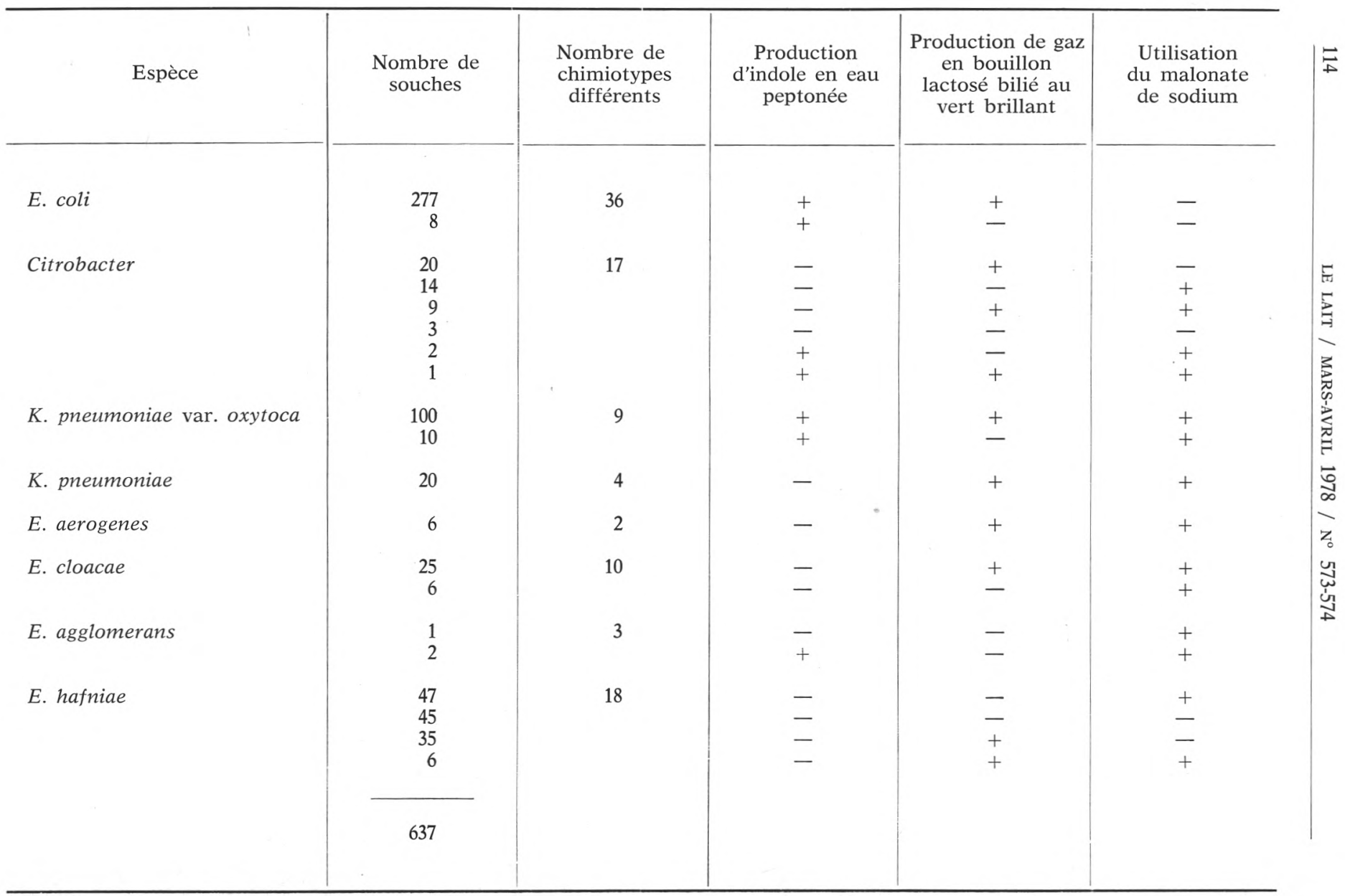


en bouillon bilié au vert brillant ne permet pas de distinguer les espèces aussi bien que le fait l'utilisation du malonate de sodium : c'est 100 p. 100 des souches d'E. coli qui sont incapables de dégrader ce composé, alors que $K$. oxytoca, ainsi que certaines variétés indologènes de Citrobacter et d'E. agglomerans, le font.

Nous constatons, de plus, que les seules souches indologènes se répartissent ainsi :

Espèces

Nombre de souches

Escherichia coli

285

K. pneumoniae var. oxytoca

110

Citrobacter

E. agglomerans

\section{DISCUSSION ET PROPOSITION}

Nous examinerons les résultats que nous avons obtenus avec les principales espèces dont nous disposions et ferons appel aux données antérieurement publiées, relatives aux souches d'origines différentes des nôtres [1, 3].

Nous avons identifié $1062 \mathrm{E}$. coli sur les 2666 colonies que nous avons isolées : 98,87 p. 100 d'entre eux sont indologènes ; c'est ce que l'on peut appeler un caractère d'une grande stabilité, tandis que Edwards et Ewing [3], relèvent, sur leur collection de 1021 souches de colibacilles, une proportion extrêmement proche de la nôtre, soit de 99,2 p. 100 d'indologènes. Par ailleurs, 100 p. 100 de nos E. coli, qu'ils soient indologènes ou non, refusent d'utiliser le malonate.

Pour $K$. oxytoca (qui constitue 6,63 p. 100 des souches retenues par nous) 100 p. 100 des 110 étudiées sont indole + , malonate + . Les Citrobacter et les E. agglomerans, indologènes, peuvent être confondus s'ils sont malonate - avec $E$. coli ; s'ils sont malonate +, avec $K$. oxytoca. Mais, la probabilité d'en isoler est très faible : il n'y a que 6,7 p. 100 de Citrobacter indologènes sur 582, d'après Edwards et Ewing [3]. Dans le cadre de notre étude, nous n'avions respectivement que 0,41 p. 100 et moins de 0,22 p. 100 de chance de trouver un Citrobacter ou un E. agglomerans indologènes.

Toutes nos souches de $K$. pneumoniae sont indole - , malonate + . Cependant, d'après Fife et al. [3], 7,5 p. 100 des types (sur 705 cultures) peuvent être malonate - . Là encore, nous avions moins de 0,5 p. 100 de chance d'en trouver.

Pour Enterobacter cloacae, 100 p. 100 des clones que nous avons testés ont utilisé le malonate de sodium, résultats que nous pouvons comparer à ceux de Barbe (100 p.100 sur un total de 68). 
Pour E. aerogenes, il n'est pas possible de donner de résultats statistiques, le nombre de souches mises en évidence étant trop peu important.

Les résultats concernant $E$. hafniae, sont variables.

Nous proposons, donc, un test simple de détermination des colonies d'E. coli : à partir d'une colonie bien isolée, ensemencer un tube d'eau peptonée et un tube de milieu au malonate de sodium*. Après incubation de $24 \mathrm{~h}$ à $37^{\circ} \mathrm{C}$, rechercher la production d'indole et l'utilisation, ou non, du malonate de soude. En pratique, toutes les colonies, à la fois, capables de produire de l'indole et refusant de métaboliser le malonate, sont des colonies de colibacilles.

La microbiologie présentant, constamment, des variations et des exceptions, même minimes, nous ne considérons comme particulièrement solide que la dualité : « indol +, malonate - », la variabilité des caractères étant nettement plus grande pour les autres coliformes que pour E. coli, qui ne peut appartenir qu'à la combinaison de caractères précitée. $\mathrm{Si}$, dans l'avenir, $L$. amalonatica venait à être isolée plus fréquemment, le test " malind " n'excluerait pas, non plus, cette espèce, dont le rôle entéropathogène est actuellement soupçonné, puisqu'elle a été isolée de selles diarrhéiques, à l'exclusion de toute autre bactérie entéropathogène [10].

Notre double épreuve ne repère pas les E. coli non indologènes, que ne mettait pas en évidence, non plus, le test de Mackenzie ; elle présente, en revanche, le grand avantage de ne pas éliminer les $E$. coli agazogènes, dont certains peuvent être entéropathogènes, comme le sérotype 0124 B 17 que nous avons étudié et à qui a été attribué il y a quelques années, une intoxication alimentaire provoquée par des fromages exportés. Ce test donne une réponse rapide ; est économique, car il ne nécessite pas une incubation à la température spéciale de $44^{\circ} \mathrm{C}$, à laquelle, d'ailleurs, certaines souches d'E. coli peuvent être inhibées.

\section{CONCLUSIONS}

Il est proposé, pour différencier des autres, les colonies de colibacilles, d'employer un test simple, fondé sur deux propriétés associées et statistiquement constantes de ces bactéries : la production d'indole, l'inutilisation du malonate de sodium. Intéressant du point de vue hygiénique, il est capable, accessoirement, de mettre en évidence $K$. oxytoca.

(Laboratoire de Microbiologie

U.E.R. des Sciences de la Vie et du Comportement 14032 Caen cedex).

\footnotetext{
* Prendre la formule précisée dans l'ouvrage de Le Minor.
} 


\section{Bibliographie}

[1] BARBE (J.) (1969). - Organisation méthodique de l'étude des caractères enzymatiques des bactéries de la tribu des Klebsielleae : application à la classification. Thèse de Doctorat en Pharmacie.

[2] Buttiaux (R.), Samaille (J.) et Pierens (Y.) 1956). - L'identification des Escherichia des eaux. Test d'Eijkman et production d'indole à $44^{\circ} \mathrm{C}$, tests I.M.V.I.C. Uréase. Ann. Inst. Past. Lille, 8, 137-149.

[3] Edwards (P.) and EwINg (W.) (1972). - Identification of Enterobacteriaceae. Burgess Publishing Company, Minneapolis, Minnesota (U.S.A.), 1 vol., 362 p.

[4] Jacouet (J.) et Coiffier (O.) (1974). - Sur la résistance aux antibiotiques des coliformes de l'intestin des animaux, et notamment des poulets. Bull. Acad. Vét., 47, 317-325.

[5] LeIfSON (E.) (1933). - The fermentation of sodium malonate as a mean of differenciating Aerobacter and Escherichia. J. Bact., 26, 329-330.

[6] Le Minor (L.) (1972). - Le diagnostic de laboratoire des bacilles à Gram négatif. I. Entérobactéries. Editions de la Tourelle, St-Mandé, 1 vol., 372.

[7] Mackenzie (E. F. W.), Taylor (E. W.) and Gilbert (W. E.) (1948). - Recent experiences in the rapid identification of Bacterium coli type I. J. Gen. Microbiol., 2, 197-204.

[8] Mehlman (I. J.), Simon (N. T.), Sanders (A. C.), Fishbein (M.), Olson (J. C.) and READ (R. B.) (1975). - Methodology for enteropathogenic Escherichia coli. J. of the A.O.A.C., 58, 283-292.

[9] Oger (C.) et Leclerc (H.) (1977). - Essai de nouveaux tests " haute température " pour la mise en évidence des coliformes fécaux et des $E$. coli dans les eaux. Microbiol., 3, 47-55.

[10] RichaRd (C.) (1975). - A propos de " nouvelles " entérobactéries : Edwardseilla tarda, Levinea malonatica et amalonatica, et Enterobacter agglomerans. Bull. Inst. Pasteur, 73, 357-381. 